\title{
Multicentric Castleman's Disease in a 21 Years Old Female : A Rare But Important Condition
}

\section{Shahed Ahmad Chowdhury ${ }^{1^{*}}$ \\ Samira Taufiq Reshma ${ }^{2}$ \\ Shanjana Islam ${ }^{3}$}

'Department of Haematology Chittagong Medical College Chittagong, Bangladesh.

${ }^{2}$ Department of Haematology Delta Health Care Ltd.

Chittagong, Bangladesh.

${ }^{3}$ Department of Pediatrics

University of Science \& Technology Chittagong (USTC) Chittagong, Bangladesh.

\section{*Correspondence to:}

\section{Dr. Shahed Ahmad Chowdhury}

Associate Professor

Department of Haematology

Chittagong Medical College

Chittagong, Bangladesh.

Mobile : +8801819318561

Email: shahed.hematology@gmail.com

\begin{abstract}
Castleman's disease, also known as angiofollicular lymph node hyperplasia, is a rare disease with two known expansion types, unicentric and multicentric, which plays a major role in determining therapy. The rare multicentric type is a lymphoproliferative disorder of unknown etiology and is characterized by various clinical manifestations and multiple organ involvement. This disease runs a more aggressive course and a poor prognosis. Optimal therapies have not been well established till now. We here report a case of rare Multicentric Castleman's Disease (MCD) in a $21 \mathrm{yrs}$ old female. She presented with slowly enlarging lymph nodes in cervical and inguinal regions which lead to a histological diagnosis of this rare condition. Its clinical features, types, relevant investigations and current treatment modalities are discussed. Though rare, early suspicion of this condition may relieve the suffering, avoid unnecessary investigations, give opportunity to choose treatment options and can save lives.
\end{abstract}

Key words : Castleman's disease; Lymph node; Hyperplasia.

\section{INTRODUCTION}

Castleman's disease is an uncommon clinicopathological entity characterized by non-neoplastic lymph node hypertrophy and histologically characterized by angiofollicular lymph node hyperplasia. Castleman et al first described it in 1956 in a group of patients with localized benign lymphadenopathy ${ }^{1}$. There are two known expansion types of this disease, unicentric and multicentric, which plays a major role in determining therapy. Because of the rarity of this disease, epidemiological data like prevalence or incidence rates are not available. The average age of patients with unicentric Castleman's disease is around 30 to 40 years of age. Patients with the multicentric form are usually around 50 to 60 years old. No dispositional age, race or sex seems to exist but the disease is widely associated with Human Herpes Virus 8 (HHV-8) and Human Immunodeficiency Virus (HIV) infection ${ }^{2}$. Most cases of Castleman's Disease represent either the hyaline vascular variant (80-90\% of cases) or the plasma cell variant $(10-20 \%)$ a small percentage present with a mixed histologic appearance ${ }^{3}$. The hyaline-vascular histology accounts for most Unicentric Castleman's Disease (UCD) cases and the plasma cell type characterizes most cases of Multicentric Castleman's Disease (MCD). UCD is typically localized, associated with minimal symptoms, and treated with local therapy alone. However, MCD is a systemic disease that commonly occurs in the setting of HIV infection and is clinically characterized by diffuse lymphadenopathy, splenomegaly, anemia, and systemic inflammatory symptoms ${ }^{4}$. MCD is primarily treated with systemic therapies ${ }^{4}$. 


\section{CASE REPORT}

A 21 years old female reported to a hospital with the complaints of fever for 1 year, which is swinging in nature, appearance and gradual enlargement of lumps under skin in cervical and inguinal regions and also with gradually distended abdomen for the same duration. She also had a history of associated anorexia, nausea, occasional vomiting and approximately $10 \mathrm{~kg}$ wt loss over last 1 year. Her past medical and family history did not suggest any specific disease.

Physical examination revealed anaemia, bilateral pedal oedema, enlarged lymph nodes in cervical and inguinal regions, the largest one was located in the cervical region, approximately $3 \times 2 \mathrm{~cm}$ in size. Per abdominal examination revealed splenomegaly about $1.5 \mathrm{~cm}$ from left costal margin. Liver was also enlarged and tender, about $2 \mathrm{~cm}$ from right costal margin in mid clavicular line. Ascites was present with shifting dullness

Blood count showed moderate anaemia $(8 \mathrm{~g} / \mathrm{dl})$ white blood cell count of $8 \times 10^{9} / \mathrm{L}$, Low normal platelet count $\left(180 \times 10^{9} / \mathrm{L}\right)$ with raised ESR (125 $\mathrm{mm}$ in $1^{\text {st }}$ hour). Liver Function Test showed SGPT $62 \mathrm{U} / \mathrm{L}$, SGOT $46 \mathrm{U} / \mathrm{L}$ and normal Prothrombin Time, but there was hypoalbuminaemia (S. Albumin $2.1 \mathrm{~g} / \mathrm{dl}$ ) and Hypergammaglobulinaemia (S. Globulin $6.3 \mathrm{gm} / \mathrm{dl}$ ). CRP was $20 \mathrm{mg} / \mathrm{dl}$. Screening for Anti HIV antibody was negative.

Ultrasonography of whole abdomen reveled hepatosplenomegaly, ascites, abdominal lymphadenopathy and hepatic Space Occupying Lesion (SOL).

FNAC of cervical lymph node showed non specific lymphadenitis but histopathological examination revealed Castleman's disease - Plasma Cell type.

\section{DISCUSSION}

Castleman's Disease is named after Benjamin Castleman, who first described the characteristic histopathological findings of angio-follicular lymph node hyperplasia in a localized lymph node region in $1954^{5}$. Clinically the disease has 2 forms, First one is localized as first described by Castleman, which is more common and another one Multicentric Castleman's Disease (MCD) with involvement of several sites, which was first described by Gaba et al. in $1972^{1}$.

The histological subtypes of Castleman's disease are as follows: Hyaline vascular variant (Unicentric in $72 \%$ of all cases) plasma cell variant (Unicentric in $18 \%$ and multicentric in $10 \%$ of all cases) mixed variant, and a plasmablastic variant of multicentric Castleman's disease ${ }^{2}$.

The localized form of the disease is mostly asymptomatic with a single site lymph node enlargement. The sites commonly involved are abdomen, peripheral lymph nodes and the mediastinum ${ }^{1}$. It is often discovered incidentally during routine examination, chest $\mathrm{X}$ rays or due to discomfort secondary to local compression ${ }^{1}$.
MCD is more common in elder male (Male/female ratio is $2.5: 1$ to $13: 1$ ). It is generally of the plasma cell type or mixed variant. MCD is a systemic disease with significant peripheral lymphadenopathy and hepatosplenomegaly, frequently with fever, night sweats, fatigue and weight loss. Abnormal laboratory findings include pancytopenia, abnormal function of liver and kidney, raised CRP, IL-6 and hypergammaglobulinemia $^{6}$. The natural history of MCD is variable. Some patients may present with indolent disease and very slow progression over months to years, while others experience a relapsing-remitting course or an acute and fulminant disease that can be fatal within weeks; the latter courses are more common in patients with HIV associated $\mathrm{MCD}^{7,8}$.

The pathogenesis of Castleman's Disease is not fully understood, however, the central roles of Interleukin (IL-6) in UCD and both IL-6 and Human Herpes Virus (HHV-8) in MCD have been well described. Patients with Castleman's Disease are at increased risk of developing lymphoma. Non-Hodgkin lymphoma has been reported in approximately $20 \%$ of patients with $\mathrm{MCD}$, as well as in some patients with $\mathrm{UCD}^{4}$.

Castleman's disease is a pathological diagnosis made by excisional biopsy of the affected lymph node tissue. In cases of deeper or less accessible tissue, core needle biopsy is preferred to fine needle aspiration, because fine needle aspirations are insensitive for both $\mathrm{UCD}$ and $\mathrm{MCD}^{4}$. Laboratory studies with blood counts and measurement of C-reactive protein, interleukin- 6 and liver function should be done. In addition, HIV and HHV-8 testing should also be performed ${ }^{2}$. In addition, there are some benign and malignant conditions, including lymphoma and thymoma, that may appear histologically similar to Castleman's disease. Therefore, immunohistologic and immunologic gene rearrangement studies of the specimens can be useful in solidifying the diagnosis. Identifying an immunophenotypically varied population of B lymphocytes with polyclonal surface and cytoplasmic immunoglobulin markers helps to confirm the diagnosis of Castleman's disease and differentiate it from lymphoma.

Localized Castleman's Disease (UCD) usually has a good prognosis and requires surgical excision of the enlarged lymph node with no further treatment. Patients generally remain asymptomatic thereafter. MCD however tends to have a variable prognosis with no established treatment consensus. A variety of combination treatments have been tried with surgical excision, chemotherapy and steroids. In patients with associated Kaposi's sarcoma, monthly combination chemotherapy (e.g. cyclophosphamide, vincristine, doxorubicin and prednisone) has been tried with limited success ${ }^{1}$. Anti-IL6 antibodies have 
shown success with systemic symptoms, as have steroids. Most treatment modalities involve immunosuppression, increasing the chance of opportunistic infections. Recent suggestions of treatment with antivirus drug, Gangciclovir or the anti-CD20 B cell monoclonal antibody, Rituximab may markedly improve outcome ${ }^{1}$.

The prognosis and outcome of the multicentric type depend on many factors, like progression rate, associated infections and co morbidities which can make the prognosis poor. There are no clear data about how long the follow-up care should be conducted $^{2}$. Appropriate follow-up care should consider type, progression rate, clinical course, response to treatment and curability of the disease. It should be planned on the basis of case merit.

\section{CONCLUSION}

Castleman's disease is a rare disorder that remains a diagnostic challenge. The aim of this case report is to revisit this very uncommon condition and emphasize the importance of histology to seal the diagnosis from other lymphoproliferative conditions. Although surgical resection remains the standard therapy for unicentric disease, the landscape for the man-agement of multicentric disease continues to evolve. Rituximab monotherapy is the current mainstay of therapy, and novel agents targeting interleukin- 6 represent exciting new additions to the treatment armamentarium. Single-agent and combination chemotherapies as well as antiviral therapy provide adjunctive support, particularly in the setting of relapsed or refractory dis-ease. The ongoing exploration of antiviral and novel strategies, such as proteasome inhibition, is heralded. The management of Castleman's disease also requires careful attention to potential concomitant infections, malignancies and associated syndromes.

\section{DISCLOSURE}

All the authors declared no competing interest.

\section{REFERENCES}

1. Iyer S, Bhatti MI, Halliday M. Castleman's disease : A case report.International Journal of Surgery Case Reports. 2010; 1: 25-26.

2. Aguilar-Rodriguez et al. Localized retroperitoneal Castleman's disease: A case report and review of the literature. Journal of Medical Case Reports. 2014; 8:93

3. Izuchukwu IS,Tourbaf K and Mahoney MC. An unusual presentation of Castleman's Disease: A case report. Journal of BMC Infectious Diseases. 2003;03.

4. Soumerai JD, Sohani AR, and Abramson JS. Diagnosis and Management of Castleman Disease. Journal of cancer control. 2014; 21:266-276.

5. Fajgenbaum DC, Rhee FV, and Nabel CS. HHV 8-negative, idiopathic multicentric Castleman disease: novel insights into biology, pathogenesis, and therapy. Blood journal. 2014;123:2924-2933.

6. Zhen-gang Yuan, Xiao-yi Dun, Yong-hua Li and Jian Hou Acta .Treatment of multicentric Castleman's Disease accompanying multiple myeloma with bortezomib: A case report. Journal of Hematology \& Oncology. 2009;2:19.

7. Bower M, Newsom-Davis T, Naresh K, et al. Clinical features and outcome in HIV associated multicentric Castleman's disease. J Clin Oncol. 2011;29(18):2481-2486.

8. Bower M. How I treat HIV-associated multicentric Castleman disease. Blood. 2010;116(22):4415-4421. 